\title{
EULAR recommendations for the role of the nurse in the management of chronic inflammatory arthritis
}

\author{
Yvonne van Eijk-Hustings, ${ }^{1}$ Astrid van Tubergen, ${ }^{2}$ Carina Boström, ${ }^{3}$ \\ Elena Braychenko, ${ }^{4}$ Beate Buss, ${ }^{5}$ José Felix, ${ }^{6}$ Jill Firth, ${ }^{7}$ Alison Hammond, ${ }^{8}$ \\ Benny Harston, ${ }^{9}$ Cristina Hernandez, ${ }^{10}$ Masa Huzjak, ${ }^{11}$ Jana Korandová, ${ }^{12}$ \\ Marja Leena Kukkurainen, ${ }^{13}$ Robert Landewé, ${ }^{14}$ Maryse Mezieres, ${ }^{4}$ \\ Marijana Milincovic, ${ }^{15}$ Antonella Moretti, ${ }^{15}$ Susan Oliver, ${ }^{16}$ Jette Primdahl, ${ }^{17,18}$ \\ Marieke Scholte-Voshaar, ${ }^{19}$ Jenny de la Torre-Aboki, ${ }^{20}$ Jennifer Waite-Jones, ${ }^{7}$ \\ Rene Westhovens, ${ }^{21}$ Heidi Andersen Zangi, ${ }^{22}$ Turid Heiberg, ${ }^{23,24}$ Jackie Hilll ${ }^{25}$
}

\begin{abstract}
- Additional data (supplementary) are published online only. To view these files please visit the journal online (http://ard.bmj.com/ content/71/1.toc).
\end{abstract}

For numbered affiliations see end of article

\section{Correspondence to} Yvonne van Eijk-Hustings, Department of Integrated Care, Maastricht University Medical Centre, Postbox 5800, 6202 AZ Maastricht, The Netherlands; yvonne.eijk.hustings@mumc.n

YVEH and AVT share first authorship for this study and $\mathrm{TH}$ and $\mathrm{JH}$ share last authorship for this study.

Received 31 May 2011 Accepted 10 September 2011 Published Online First 28 October 2011

\begin{abstract}
Objectives The authors aim to develop European League Against Rheumatism recommendations for the role of the nurse in the management of patients with chronic inflammatory arthritis, to identify a research agenda and to determine an educational agenda.

Methods A task force made up of a multidisciplinary expert panel including nurses, rheumatologists, occupational therapist, physiotherapist, psychologist, epidemiologist and patient representatives, representing 14 European countries, carried out the development of the recommendations, following the European League Against Rheumatism standardised operating procedures. The task force met twice. In the first meeting, the aims of the task force were defined, and eight research questions were developed. This was followed by a comprehensive, systematic literature search. In the second meeting, the results from the literature review were presented to the task force that subsequently formulated the recommendations, research agenda and educational agenda.
\end{abstract}

Results In total, 10 recommendations were formulated. Seven recommendations covered the contribution of nurses to care and management: education, satisfaction with care, access to care, disease management, psychosocial support, self-management and efficiency of care. Three recommendations focused on professional support for nurses: availability of guidelines or protocols, access to education and encouragement to undertake extended roles. The strength of the recommendations varied from $A$ to $C$, dependent on the category of evidence (1 $A-3)$, and a high level of agreement was achieved. Additionally, the task force agreed upon 10 topics for future research and an educational agenda. Conclusion 10 recommendations for the role of the nurse in the management of chronic inflammatory arthritis were developed using a combination of evidence-based and expert consensus approach.

\section{INTRODUCTION}

In rheumatology, registered nurses often act as the interface between patients and other members of the multidisciplinary team. As a result of new treatment regimens and organisational developments, the role of the nurse is undergoing great change. However, there are large differences across countries and regions. In several European countries, rheumatology as a nursing specialty does not exist, whereas in other countries, it has developed into a recognised specialty with nurses undertaking advanced and extended roles. ${ }^{1}$ These include selfmanagement support, patient education and counselling, intra-articular injections, recommendation for and the prescription of drug treatments, referral to other health professionals, hospital admission of patients, manning telephone advice lines and monitoring disease-modifying and biological treatments. $^{2-8}$ Nurse-led clinics have been established, and their effectiveness has been shown to bring added value to patients' outcomes ${ }^{9-11}$ at a lower cost. ${ }^{12}$ While some countries have accepted that interventions undertaken by nurses are essential to effectively tackle the challenges of chronic illness in an economic and integrated fashion, this concept has not developed everywhere.

The aim of the present study was to evaluate the currently available literature according to the European League Against Rheumatism (EULAR) standardised operating procedures in order to provide recommendations for the role of the nurse in the management of chronic inflammatory arthritis (CIA). ${ }^{13}$ This evidence-based approach was complemented by an expert consensus approach.

\section{METHODS}

A multidisciplinary task force made up of 15 nurses, a rheumatologist, an occupational therapist, a psychologist, a physiotherapist, two patient representatives and a research fellow, representing several European countries, met twice under the leadership of two conveners and a rheumatologist/ clinical epidemiologist.

During the first meeting, the task force formulated eight research questions. These questions served as a guide to the systematic literature review (SLR) and subsequently as the basis for the recommendations. The term 'CIA' was confined to rheumatoid arthritis (RA), ankylosing spondylitis and psoriatic arthritis, thereby excluding other systemic inflammatory conditions (eg, systemic lupus erythematosus) and non-inflammatory disorders (eg, fibromyalgia).

The target population for the recommendations was chosen to be healthcare professionals working 
in the field of rheumatology (rheumatologists, nurses and other disciplines), patients and policymakers.

After translation of the research questions into relevant search terms (online supplementary appendix 1), an extensive SLR of MEDLINE, Embase, Cochrane CENTRAL, Cumulative Index to Nursing and Allied Health Literature and PsycINFO was performed in August 2010. Two main search terms-'inflammatory arthritis' and 'nurse'-were searched for in titles, keywords or full texts using MeSH (Medical Subject Headings) headings, subheadings, thesaurus or free text words and truncation symbols. No limitations with regard to publication type, research type, language or time period were applied. Selected titles and abstracts were screened independently for eligibility by AvT and YvE-H. Eligibility criteria were 'inflammatory arthritis', 'interventions undertaken by nurses' and 'relevant outcomes to answer the research questions'. The selection was shared with the other members of the task force, and suggestions and comments by the experts complemented the result of the SLR. Additionally, abstracts from American College of Rheumatology and EULAR meetings (2008-2010) were searched (selection procedure is shown in online supplementary appendix 2).

During the second meeting, the formulation of the recommendations was discussed by the entire group until a consensus was reached. The recommendations were graded based on the level of evidence of the literature found (online supplementary appendix 3$){ }^{13}$ This was sent to each participant for final approval and voting on a scale from 0 to 10 . Finally, the task force agreed upon the formulation of a research agenda and an educational agenda.

\section{RESULTS}

In total, 54 studies met the inclusion criteria (online supplementary appendix 4). The selection comprised 1 meta-analysis, 8 randomised controlled trials (RCTs), 2 controlled clinical trials, 9 quasi-experimental studies and 34 descriptive studies. As the majority of the studies were descriptive, it was acknowledged that there was a high risk of bias.

Table 1 shows the 10 recommendations with a level of evidence ranging from $1 \mathrm{~A}$ to 3 . The strength of recommendations varied from $\mathrm{A}$ to $\mathrm{C}$, and a high level of expert agreement was achieved.

\section{Recommendations}

All recommendations relate to care delivered by registered nurses with a specific training in rheumatology. This care includes the monitoring of disease consequences on the level of daily activities, participation and psychosocial consequences and, increasingly, the monitoring of disease activity, drug treatment and drug side effects.

The task force judged that the level of evidence for the role of the nurse is far greater in the management of RA than in the management of ankylosing spondylitis and psoriatic arthritis, and therefore, the recommendations should be regarded as points to consider for patients with these conditions.

The order of the recommendations follows the discussion in the task force. The first three recommendations are formulated from the patients' perspective, and the other recommendations are formulated from the nurses' perspective.

1. Patients should have access to a nurse for education to improve knowledge of CIA and its management throughout the course of their disease.

Patient education is defined as a planned process aiming to improve coping strategies and increase self-care abilities. ${ }^{14} 15$
A statistically significant increase in the patient's knowledge of the disease process, treatment strategies (eg, drug treatment), physiotherapy and self-management strategies (eg, joint protection techniques) was found in patients with RA who were educated during monitoring ${ }^{10}$ or who received a specific educational programme from nurses. ${ }^{16-18}$ Moreover, statistically significant greater levels of knowledge were found in patients monitored and educated by a nurse compared to patients monitored by doctors. ${ }^{9}$

Involvement in disease management allows nurses to offer timely education to newly diagnosed and established patients. In addition to information about their disease and treatments, education should address risk factors for comorbidities, such as cardiovascular problems. ${ }^{19}$ Nurses can also play an important role in educating patients about the principles of 'treat to target $^{\prime 20}$ in order to enhance adherence. Overall, the literature demonstrates that education by nurses improves the patients' knowledge of their disease and disease-related issues.

2. Patients should have access to nurse consultations in order to experience improved communication, continuity and satisfaction with care.

Satisfaction with care is considered an indicator of the quality of care..$^{21}$ The majority of the studies showed statistically significant increased satisfaction with information, empathy, technical quality and attitude of the professional, as well as access to care in patients with RA when monitored by a nurse compared to monitoring by doctors or other health professionals. ${ }^{9} 1022-24$ There were no differences in patient satisfaction after intraarticular injections given by either a nurse or a doctor. ${ }^{25}$ Patients valued nurses' communication skills and nursing care in terms of advice to use assistive devices, referral to other members of the multidisciplinary team and education. ${ }^{26}{ }^{27}$ Quality and continuity of care in nurse-led clinics were perceived as good. ${ }^{28}$ Holistic care and patient-centred information were found to contribute to patient satisfaction. ${ }^{29}$

However, some studies report conflicting evidence. One study did not find statistically significant changes in patients' satisfaction after monitoring by a nurse practitioner..$^{30}$ In another study, patients' satisfaction with multidisciplinary team care was found to be significantly higher than care coordinated by a clinical nurse specialist. ${ }^{31}$

Nurses tend to be accessible to patients and can facilitate access to services. Continuity of care provides the opportunity to establish a confidential and long-standing patient-professional relationship that is considered to be important by patients. Despite some conflicting evidence, the task force concluded that disease monitoring or follow-up care by nurses enhances patients' perceptions of care.

3. Patients should have access to nurse-led telephone services to enhance continuity of care and to provide ongoing support.

The unpredictable, fluctuating nature of rheumatic diseases means that rapid access to advice is of utmost importance for patients. Telephone helplines were found to support accessibility to care. ${ }^{32}$ Patients perceived that telephone helplines support enhanced continuity of care and provided them with access to a knowledgeable practitioner who would often be the first point of contact at times of need. Mostly, patients stated that they would call again if needed. ${ }^{32-35}$ The contents of the service that was offered by the helplines were not clearly described in all studies, and its focus varied. Some services provided regular follow-up, ${ }^{33}$ while others focused on support by answering disease-related questions or worries that might avoid unnecessary consultations with general practitioners. ${ }^{32}$ There were 
Table 1 Recommendations for rheumatology nursing management of CIA

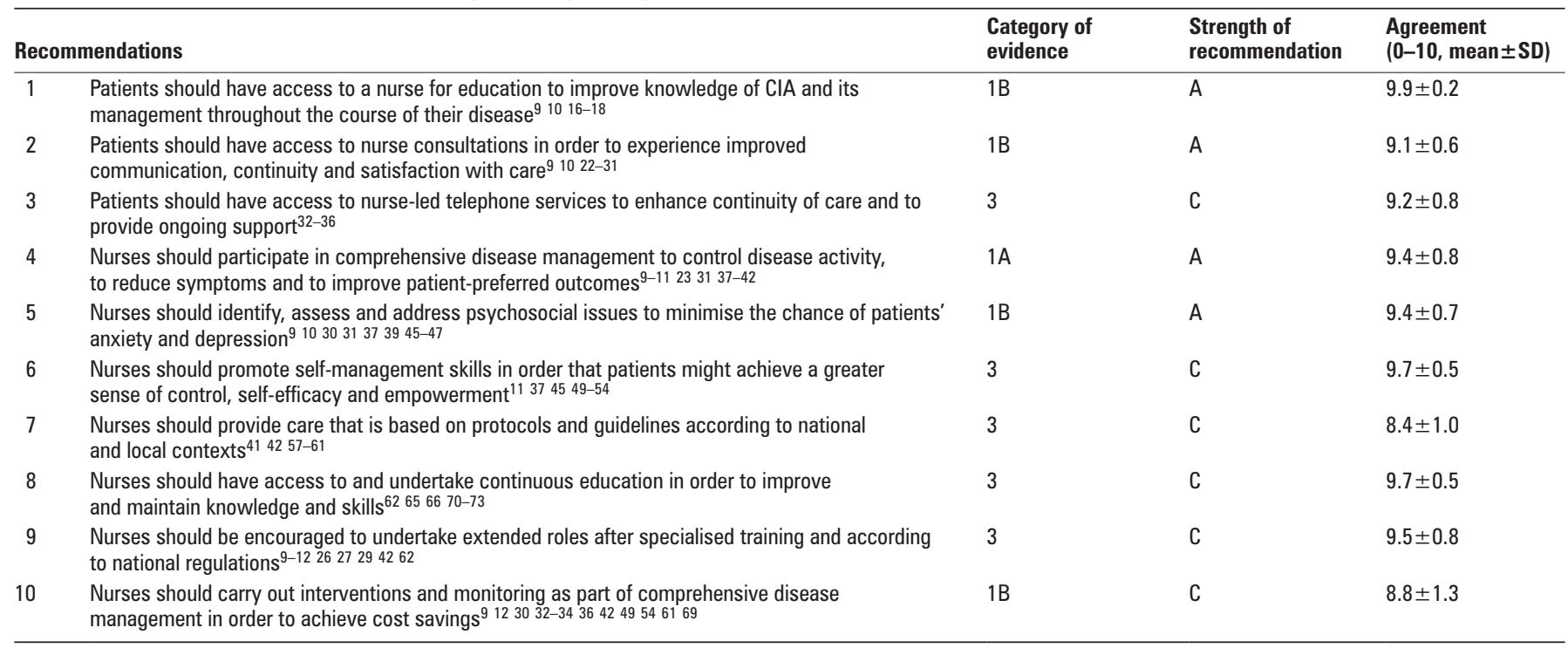

CIA, chronic inflammatory arthritis.

also examples of helplines that focused specifically on triage to identify patients that required fast-track clinical assessment. ${ }^{36}$ In countries where telephone services are not available, information and support are sometimes given by email. In general, both means of support contribute to enabling patient accessibility and appropriate care.

4. Nurses should participate in comprehensive disease management to control disease activity, to reduce symptoms and to improve patient-preferred outcomes.

Nurses have increasingly combined providing support to patients in a broad spectrum of disease-related problems, such as psychosocial problems and limitations in participation, with disease monitoring. Several studies showed that nurseled care results in equivalent suppression of disease activity in patients with RA, in comparison with medical care..$^{9-11} 2331$ 37-39 Moreover, appropriately trained nurses were able to detect early arthritis, ${ }^{40}$ make referrals, determine necessary interventions and change medications. ${ }^{41} 42$

Patients with RA also perceived statistically significant less pain ${ }^{9}$ and fatigue when monitored by nurses, compared to doctors. ${ }^{10}$ Research showed that these symptoms contribute to patients' perceptions of disease impact, and therefore, patients consider them as preferred outcomes in clinical trials. ${ }^{43}$

There is evidence that nurses can manage CIA appropriately, that they can contribute to symptom control and that they can play a role in the early detection of arthritis.

5. Nurses should identify, assess and address psychosocial issues to minimise the chance of patients' anxiety and depression.

The psychosocial impact of CIA is considerable, and anxiety and depression are both well-known comorbidities in RA. ${ }^{44}$ One study found a statistically significant reduction in anxiety and depression in patients with RA after monitoring by a nurse. ${ }^{9}$ Others showed equivalent reductions in anxiety and depression in patients receiving nurse-led or medical care. ${ }^{10} 37$

It has been shown that cognitive-behavioural interventions provided by a nurse can statistically significantly improve the emotional well-being in patients with RA. ${ }^{45}$ Moreover, quality of life was comparable between patients receiving either nurse-led or multidisciplinary team care. ${ }^{30} 3139$ Psychosocial adjustment, which is considered as overall adaptation to the impact of RA, was comparable in patients monitored by either a nurse or a doctor, but patients monitored by a nurse reported supplementary increased social activities. ${ }^{46}$ Patients valued the opportunity to discuss the wider implications of their condition with a nurse. ${ }^{47}$ Counselling in psychological issues is considered important, but undertaking this role depends strongly on the level of the problems, the skills of the nurse and the ability to access other sources of support when required-for example, the availability of psychologists in assisting with these tasks. Therefore, the task force considered identifying problems and referring to other professionals when needed as key components of nursing care.

6. Nurses should promote self-management skills in order that patients might achieve a greater sense of control, self-efficacy and empowerment.

Self-efficacy refers to the belief that one can successfully execute the behaviour required to attain certain goals and, thus, to feel in control. ${ }^{48}$ Research shows that nurses are likely to contribute to increased perceived control, levels of self-efficacy and empowerment. Patients reviewed by a clinical nurse specialist in a drug-monitoring clinic perceived clinically relevant increased perception of control. ${ }^{11}$ After a cognitive-behavioural intervention provided by nurses, patients demonstrated a statistically significant increase in personal coping resources, such as competency beliefs and decrease of helplessness. ${ }^{45}$ Nurse-led management, information and support have been shown to increase self-efficacy beliefs and self-reliance of patients ${ }^{49} 50$ as well as to contribute to patient empowerment. ${ }^{50-52}$

However, some studies were unable to demonstrate any changes in lifestyle, self-management behaviour or self-efficacy after interventions undertaken by nurses. ${ }^{37} 5354$

Support with regard to self-management comprises all actions that encourage patients to manage their own disease, and this is a task for all members of the multidisciplinary team. As selfmanagement support is multifaceted, the task force considered this as an important role of the nurse, rather than a single intervention undertaken by nurses.

7. Nurses should provide care that is based on protocols and guidelines according to national and local contexts.

Guidelines provide research-based options for decisions, whereas protocols describe steps to be taken to reduce variation in the treatment of patients. ${ }^{55}$ Guidelines and protocols are essential for all healthcare professionals to ensure safe and 
high-quality care. Often, these guidelines and protocols will be adapted to a national or local context. ${ }^{56}$

It has been demonstrated that structured implementation material supported nurses in the guidance of patients with a complex treatment regimen. Furthermore, nurses' perceived capability for guidance statistically significantly increased after an educational session. ${ }^{57}$ Guidelines have been found to support nurses' clinical decision-making skills with regard to assessment and treatment, ${ }^{58}$ referral, ${ }^{42}$ supplementary prescription, ${ }^{41}$ and therefore contribute to evidence-based nursing ${ }^{59}$ and holistic care. ${ }^{41} 58$ Protocols have been found to support continuity and safety of care in terms of immediate and appropriate adjustments of treatment ${ }^{41} 4260$ and enabled nurses to discriminate between early arthritis and other conditions. ${ }^{61}$

However, standardised care should be implemented alongside national and regional regulations yet enable an individualised, patient-centred approach. Overall, guidelines and protocols are likely to support nurses in providing evidence-based care.

8. Nurses should have access to and undertake continuous education in order to improve and maintain knowledge and skills.

The literature demonstrates that nurses undertake a wide variety of interventions, including self-management support, education, counselling, drug monitoring, drug prescription and administration of intra-articular injections. ${ }^{62-69}$ In a survey undertaken in the UK, the majority of the nurses felt confident in their abilities to undertake these tasks. ${ }^{65}$ The self-confidence of nurses is supported by knowledge of rheumatic diseases and their treatment, skills in relation to education, counselling and training, collaboration with other health professionals and manual skills. ${ }^{70}$ Furthermore, nurse education needs to be tailored to reflect research findings if nurses are to maintain and enhance their knowledge and skills appropriately. ${ }^{62} 70$ Studies have demonstrated that the contents of consultations and issues for patient education and counselling are dependent on the educational level of nurses. ${ }^{71-73}$ However, it has been reported that knowledge and skills do not appear to be sufficiently covered by basic and advanced training at present. ${ }^{66}$

Educational opportunities in nursing vary to a large extent if an educational curriculum is not defined accurately. Given the complexity of the tasks and activities that are performed by nurses, ongoing access to well-defined education on a basic, advanced and extended level is needed.

9. Nurses should be encouraged to undertake extended roles after specialised training and according to national regulations.

Increasingly, nursing care expands from a more basic level to an advanced or an extended level. The rationale for these developments comes from several perspectives. From the patient's perspective, holistic care, patient-centred information and communication skills of nurses are appreciated, and improved outcomes such as knowledge, satisfaction and physical and psychological symptoms were found. ${ }^{9-11} 26272942$ From the professional's perspective, job satisfaction is enhanced by greater autonomy and by optimal use of nurses' qualities and skills. ${ }^{42} 62$ From an organisational perspective, advanced and extended roles may prevent fragmentation of care and promote efficiency and accessibility. ${ }^{12}{ }^{62}$ Key components for achieving extended roles include performing outpatient procedures, prescribing drugs and treatment and taking a lead in the organisation of local health services. ${ }^{62}$

However, some concerns about extended nursing roles have been raised among members of the medical and the nursing professions about being 'placebo-doctors' or 'second-rate doctors'. ${ }^{26}{ }^{62}$ If role development is patient-focused, aiming to meet patients' identified needs and to improve overall patient care, the extended role of the nurse should be regarded as a complement to the medical role and not only as a substitution of medical tasks.

The competencies and skills of the nurse should be considered and optimised to further improve patient care, to enhance and mobilise nursing competency and to improve efficiency of care.

10. Nurses should carry out interventions and monitoring as part of comprehensive disease management in order to achieve cost savings.

Innovative nurse-led care has advanced to increase efficiency of care. Coordinated care by a clinical nurse specialist was shown to be statistically significantly cost-effective, compared with multidisciplinary inpatient or day-patient care. ${ }^{12}$ Additional costs for a nurse practitioner to a medical team were found to be partially compensated by taking over tasks from other team members. ${ }^{30}$ Moreover, nurse-led monitoring led to decreased medical referrals ${ }^{42}$ or decreased the length of stay in a rehabilitation programme for patients with $\mathrm{RA},{ }^{49}$ all pointing to cost savings. Furthermore, rheumatology telephone helplines are likely to contribute to cost savings by decreasing the number of unnecessary doctor consultations ${ }^{32} 3436$ and by reducing follow-up waiting time. ${ }^{33}$ Accessibility of care also improved with the nurse being able to discriminate different categories of early arthritis ${ }^{61}$ Recent preliminary data suggest a positive cost-benefit of the role of nurse specialists by preventing unscheduled care and hospital admissions. ${ }^{69}$

However, compared to usual care from a rheumatologist, patients seen by a nurse were more frequently referred to occupational therapists, and this difference was statistically significant. ${ }^{954}$ It is arguable whether this phenomenon is a consequence of the greater emphasis that nurses placed on joint protection and improving functioning in daily living 9 and, therefore, could be considered as quality improvement of care rather than increase in costs. ${ }^{54}$ There is a need for high-quality economic analyses in future research.

\section{Research agenda}

In addition to the developed recommendations, a research agenda and an educational agenda were formulated. The research agenda highlights where there is weak or lacking evidence to further optimise the role of the nurse in the management of CIA and is shown in box 1 .

\section{Educational agenda}

The educational agenda was defined to support educational opportunities for nurses and to guarantee quality in nursing care (shown in box 2).

\section{DISCUSSION}

Nurses are the largest group of healthcare professionals, and their role development in the provision of team care for patients with rheumatic diseases follows a worldwide tendency to more proactive, evidence-based care for patients with chronic disorders with nurses fulfilling extended roles. ${ }^{745}$ The role of nurses in the management of CIA appeared to differ greatly between countries and across regions due to their educational level, training and expertise, as well as to national and regional regulations and contexts and funding issues related to overall healthcare provision.

Evidence for the additional value of nurses was most obvious when disease monitoring by nurses was combined with support 


\section{Box 1 Research agenda}

To study the contribution of the nurse in improving access to care and in facilitating the effective utilisation of care provided by members of the multidisciplinary team

To study the role of nurses in optimising 'treat to target' in early disease

To study the contribution of the nurse in improving patient-preferred outcomes

To compare the different components of nursing care in each European country in relation to knowledge and competencies To perform cost-effectiveness studies across different European countries, on the role of the nurse in basic and advanced practice To study the long-term effects of interventions by a nurse on quality of life, psychosocial and general well-being

To study the contribution of the nurse in improving self-management and self-efficacy

To study the impact of interventions by a nurse on the patient's employment status and social participation

To define the contribution of the nurse in the prevention of comorbidities

To study the recommendations in different patient populations including ankylosing spondylitis and psoriatic arthritis

for patients in a broad spectrum of disease-related problems. ${ }^{9-11}$ However, roles, tasks and qualifications should be clearly described in frameworks to practice that include protocols and guidelines.

Nurses tend to be accessible for patients. Given their qualities and skills with regard to coordination of care, ${ }^{31} 39$ they may facilitate increased access to multidisciplinary team care. In practice, however, some tasks may be provided by other health professionals, depending on local accessibility and competency. It is arguable whether these tasks have the same quality when provided by different health professionals. This study explicitly deals with nurses, because this profession is often not clearly visible in multidisciplinary team care, but their role should be considered in the context of care delivery of other healthcare providers and an active role of patients. By their continuous presence, nurses can identify and communicate specific areas that can be addressed by other members of the multidisciplinary team.

Ten recommendations for the role of the nurse in the management of patients with CIA were developed. There are some limitations to these recommendations that need to be addressed.

First, the task force decided to include all types of studies that could give insight in nursing care, as only a limited number of RCTs exist, and RCTs alone may not adequately cover the topic. ${ }^{76} 77$ Qualitative studies provide important insight in patients' individual needs, functional limitations and the extent to which different types of care meet those needs, ${ }^{78}$ all of which may improve quality of care..$^{21} 2879$ However, these studies are rated with a lower level of evidence than RCTs, and subsequently, there is a high risk of bias. By including the additional and clinically relevant information retrieved from these non-controlled studies and combining this with a consensus approach, the task force considered that this has ultimately led to more inclusive recommendations.

Second, the task force intended to formulate strong statements that were useful in emphasising the role of the rheumatology

\section{Box 2 Educational agenda}

To develop a competency framework for nurses To develop educational nursing programmes at the basic and advanced level

nurse throughout Europe. The opinion of the task force members, all representatives from nurses as well as other relevant parties, was considered of additional value in deciding on the strength of the recommendations. This may have contributed to an overestimation of the strength of recommendations. However, the high level of agreement within the task force supported the method used. Agreement with the recommendations by nurses, rheumatologists, healthcare professionals in daily practice and patients will soon be validated simultaneously with dissemination of the recommendations.

The research agenda will support future directions of nursing research, and it is important that high-quality studies, with clear descriptions of nursing roles and interventions, will be conducted as the role of the nurse in care is currently often not clearly stated in studies. ${ }^{80}$ Given the research issues and themes of importance, qualitative and quantitative insights into nursing care are needed.

The educational agenda will support access to high-quality education for nurses; will enhance clarity about knowledge, skills and competencies required by nurses, and will reduce diversity of the nursing role within and between countries.

The dissemination and implementation of the recommendations will need overall support from EULAR. Implementing these recommendations, including education, demands a clear implementation strategy in which barriers will need to be assessed. The extent to which such barriers can be addressed will be influenced by national and local regulations.

In conclusion, this study provides 10 evidence-based and expert opinion-based recommendations on the role of the nurse in the management of CIA. These recommendations provide a basis for emphasising and optimising rheumatology nursing care in order to contribute to a more standardised level of professional nursing across Europe.

Contributors During the development of the recommendations, the steering group discussed authorship, and the authors decided to include all participants who attended at least one meeting of the task force. This was also discussed with the members of the task force. All 26 authors contributed to the development process of the recommendations, drafting of the article and final approval of the version of the study to be published.

Acknowledgements The authors thank Sofia Ramiro (PhD candidate) and Wies Limpens (Maastricht University Library) for their contribution to the systematic literature review.

Funding This project was financially supported by the European League Against Rheumatism (EULAR).

\section{Competing interests None.}

Provenance and peer review Not commissioned; externally peer reviewed.

Author affiliations ${ }^{1}$ Department of Integrated Care, Maastricht University Medica Centre, Maastricht, The Netherlands

${ }^{2}$ Department of Internal Medicine, Division of Rheumatology, Maastricht University Medical Center, Maastricht, The Netherlands

${ }^{3}$ Department of Neurobiology, Care Sciences and Society, Division of Physiotherapy, Karolinska Institutet, Stockholm, Sweden

${ }^{4}$ Service de Recherche Clinique en Rhumatologie B du Professeur M Dougados, Hôpital Cochin, Paris, France

${ }^{5}$ Rheumatology, Charité Universitätsmedizin, Berlin, Germany

${ }^{6}$ Rheumatology Unit, Main Military Hospital, Lisbon, Portugal

7Pennine MSK Partnership Ltd Integrated Care Centre, Oldham, UK 
${ }^{8}$ Centre for Health, Sport and Rehabilitation Research, University of Salford, Salford, UK ${ }^{9}$ West Lodge, Blofield, UK

${ }^{10}$ Spanish League of Arthritis Patient Associations, Madrid, Spain

${ }^{11}$ Department of Rheumatology, University Medical Centre Ljubljana, Ljubljana,

Slovenia

${ }^{12}$ Institute of Rheumatology, Prague, Czech Republic

${ }^{13}$ Finnish Society of Rheumatology Nurses (FSRN), Heinola, Finland

${ }^{14}$ Department of Internal Medicine, Division of Rheumatology, Academic

Medical Centre/University of Amsterdam and Atrium Medical Centre, Heerlen,

The Netherlands

${ }^{15}$ Clinica Reumatologica, Università Politecnica delle Marche, Ospedale C Urbani, Jesi Ancona, Italy

${ }^{16}$ Minerva Health Centre, Preston, UK/Barnstaple, UK

${ }^{17}$ King Christian X's Hospital for Rheumatic Diseases, Graasten, Denmark

${ }^{18}$ Institute of Regional Health Services Research, University of Southern Denmark, Odense, Denmark

${ }^{19}$ Dutch League of Arthritis Patient Associations, Amersfoort, The Netherlands

${ }^{20}$ Department of Rheumatology, Alicante General and University Hospital, Alicante, Spain

${ }^{21}$ Department of Rheumatology, University Hospital Leuven, Leuven, Belgium

${ }^{22}$ National Resource Center for Rehabilitation in Rheumatology (NRRK).

Diakonhjemmet Hospital, Oslo, Norway

${ }^{23}$ Department for Research and Education, Oslo University Hospital, Oslo, Norway

${ }^{24}$ Department of Rheumatology, Lovisenberg Diaconal College, Diakonhjemmet Hospital, Oslo, Norway

${ }^{25}$ Academic and Clinical Unit for Musculoskeletal Nursing (ACUMeN), Academic Section of Musculoskeletal Disease, Leeds Institute of Molecular Medicine, Leeds, UK

\section{REFERENCES}

1. Carr A. Defining the extended clinical role for allied health professionals in rheumatology. ARC Conference Proceedings N012, Arthritis Research Campaign, 2001, Chesterfield, UK.

2. Bands VE. Nursing care of patients with rheumatoid arthritis. John Hopkins Advanced Studies in Nursing 2007;5:23-31.

3. Daul P, Grisanti J. Monitoring response to therapy in rheumatoid arthritis— perspectives from the clinic. Bull NYU Hosp Jt Dis 2009;67:236-42.

4. Hill J, Hale C. Clinical skills: evidence-based nursing care of people with rheumatoid arthritis. Br J Nurs 2004;13:852-7.

5. Oliver S. Improving long-term outcomes for rheumatoid arthritis: evidence-based care. Prim Health Care 2010;20:30-9.

6. Palmer D, El Miedany Y. Biological nurse specialist: goodwill to good practice. Br J Nurs 2010;19:477-80.

7. Thwaites C, Ryan S, Hassell A. A survey of rheumatology nurse specialists providing telephone helpline advice within England and Wales. Rheumatology (Oxford) 2008; $47: 522-5$.

8. Meadows A, Sheehan NJ. Prescribing and injecting: the expanding role of the rheumatology nurse. Musculoskeletal Care 2005;3:176-8.

9. Hill J, Bird HA, Harmer R, et al. An evaluation of the effectiveness, safety and acceptability of a nurse practitioner in a rheumatology outpatient clinic. Br J Rheumatol 1994:33:283-8.

10. Hill J, Thorpe R, Bird H. Outcomes for patients with RA: a rheumatology nurse practitioner clinic compared to standard outpatient care. Musculoskeletal Care 2003; 1:5-20.

11. Ryan S, Hassell AB, Lewis $\mathrm{M}$, et al. Impact of a rheumatology expert nurse on the wellbeing of patients attending a drug monitoring clinic. J Adv Nurs 2006;53:277-86.

12. van den Hout WB, Tijhuis GJ, Hazes JM, et al. Cost effectiveness and cost utility analysis of multidisciplinary care in patients with rheumatoid arthritis: a randomised comparison of clinical nurse specialist care, inpatient team care, and day patient team care. Ann Rheum Dis 2003;62:308-15

13. Dougados M, Betteridge N, Burmester GR, et al. EULAR standardised operating procedures for the elaboration, evaluation, dissemination, and implementation of recommendations endorsed by the EULAR standing committees. Ann Rheum Dis 2004; 63:1172-6.

14. Hirano PC Laurent DD, Lorig K. Arthritis patient education studies, 1987-1991: a review of the literature. Patient Educ Couns 1994:24:9-54

15. Lorig K, Konkol L, Gonzalez V. Arthritis patient education: a review of the literature. Patient Educ Couns 1987:10:207-52.

16. Burma MR, Rachow JW, Kolluri S, et al. Methotrexate patient education: a quality improvement study. Arthritis Care Res 1996;9:216-22.

17. Grahame $\mathbf{R}$, West J. The role of the rheumatology nurse practitioner in primary care: an experiment in the further education of the practice nurse. Br J Rheumato 1996; $35: 581-8$.

18. Rodriguez LC, Bilbao A, Naranjo A, et al. Patient education in rheumatoid arthritis: its influence in the disease outcome. Rev Esp Reumatol 1996;23:40-8.
19. Peters MJ, Symmons DP, McCarey D, et al. EULAR evidence-based recommendations for cardiovascular risk management in patients with rheumatoid arthritis and other forms of inflammatory arthritis. Ann Rheum Dis 2010; 69:325-31

20. Smolen JS, Aletaha D, Biilsma JW, et al. Treating rheumatoid arthritis to target: recommendations of an international task force. Ann Rheum Dis 2010;69:631-7.

21. Jacobi CE, Boshuizen HC, Rupp I, et al. Quality of rheumatoid arthritis care: the patient's perspective. Int J Qual Health Care 2004;16:73-81.

22. Arthur $\mathbf{V}$, Clifford $\mathrm{C}$. Rheumatology: a study of patient satisfaction with follow-up monitoring care. J Clin Nurs 2004;13:325-31.

23. Koksvik H, Magnussen A, Zangi H. Patient satisfaction with nurse-led care in a rheumatology outpatient clinic. Two year follow-up of a randomised controlled trial (abstract). Ann Rheum Dis 2009;68(Suppl 3):770.

24. van Eijk Y, Boonen A, Schulpen G, et al. Safety and patient satisfaction of infliximab administration in an extramural setting supervised by a rheumatology specialist nurse. Ann Rheum Dis 2006:65:276.

25. Edwards $\mathbf{J}$, Hassell A. Intra-articular and soft tissue injections by nurses: preparation for expanded practice. Nurs Stand 2000;14:43-6.

26. Hill J. Patient evaluation of a rheumatology nursing clinic. Nurs Times 1986;82:42-3

27. Wright $\mathbf{V}$, Hopkins R. Patients' perceptions of staff in a department of rheumatology. Br J Rheumatol 1990:29:374-6.

28. Temmink D, Hutten JB, Francke AL, et al. Quality and continuity of care in Dutch nurse clinics for people with rheumatic diseases. Int J Qual Health Care 2000;12:89-95

29. Mäkeläinen $\mathbf{P}$, Vehviläinen-Julkunen $\mathrm{K}$, Pietilä AM. Rheumatoid arthritis patient education: RA patients' experience. J Clin Nurs 2009;18:2058-65

30. van der Sluis CK, Datema $\mathrm{L}$, Saan I, et al. Effects of a nurse practitioner on a multidisciplinary consultation team. J Adv Nurs 2009;65:625-33.

31. Tijhuis GJ, Zwinderman AH, Hazes JM, et al. A randomized comparison of care provided by a clinical nurse specialist, an inpatient team, and a day patient team in rheumatoid arthritis. Arthritis Rheum 2002;47:525-31.

32. Hughes RA, Carr ME, Huggett A, et al. Review of the function of a telephone helpline in the treatment of outpatients with rheumatoid arthritis. Ann Rheum Dis 2002;61:341-5

33. Hennell S, Spark E, Wood B, et al. An evaluation of nurse-led rheumatology telephone clinics. Musculoskeletal Care 2005;3:233-40.

34. Newton P, Buckley H, Ali H, et al. Rheumatology telephone helpline and its role in provision of care to patients (abstract). Ann Rheum Dis 2008;67(Suppl II):583.

35. Wylie E, McAteer C, Collins D. An audit of patient and staff views of a rheumatology telephone helpline service (1888). Presented at American College of Rheumatology 2008 Annual Scientific Meeting. San Fransico, 2008

36. Leung ML. The usefulness of rheumatology nurse managed telephone helpline in enhancing patient care and reducing unnecessary early clinic attendance (abstract). Ann Rheum Dis 2008;67(Suppl II):669.

37. Ndosi M. Hill J, Hale C. Is nurse-led care for RA patients clinically effective? (abstract). Arthritis Rheum 2009;60(Suppl 10):1981.

38. Ndosi M, Vinall K, Hale C, et al. Does nurse-led care have an effect in RA disease activity? A meta-analysis (abstract). Ann Rheum Dis 2010;69(Suppl 3):722.

39. Tijhuis GJ, Zwinderman AH, Hazes JM, et al. Two-year follow-up of a randomized controlled trial of a clinical nurse specialist intervention, inpatient, and day patient team care in rheumatoid arthritis. J Adv Nurs 2003;41:34-43.

40. Gormley GJ, Steele WK, Gilliland A, et al. Can diagnostic triage by general practitioners or rheumatology nurses improve the positive predictive value of referral to early arthritis clinics? Rheumatology (Oxford) 2003:42:763-8.

41. Hennell S, Spark E, Wood B, et al. Supplementary prescribing for rheumatology patients: an evaluation. Nurse Prescriber 2005;2. http://www.nurse-prescriber.co.uk/ Articles/SuppPx_Rheumatology.htm \#Cite (accessed 21 September 2011).

42. Ryan S. Nurse-led drug monitoring in the rheumatology clinic. Nurs Stand 1997;11:45-7

43. Gossec L, Dougados M, Rincheval N, et al. Elaboration of the preliminary Rheumatoid Arthritis Impact of Disease (RAID) score: a EULAR initiative. Ann Rheum Dis 2009;68:1680-5.

44. Hill CL, Gill T, Taylor AW, et al. Psychological factors and quality of life in arthritis: population-based study. Clin Rheumatol 2007;26:1049-54.

45. Sinclair VG, Wallston KA, Dwyer KA, et al. Effects of a cognitive-behavioral intervention for women with rheumatoid arthritis. Res Nurs Health 1998;21:315-26.

46. Earle JR, Perricone PJ, Maultsby DM, et al. Psycho-social adjustment of rheumatoid arthritis patients from two alternative treatment settings. J Rheumatol 1979;6:80-7.

47. Ryan S. Rheumatology. Sharing care in an outpatient clinic. Nurs Stand 1995; 10:23-5

48. Bandura A. Self-efficacy: toward a unifying theory of behavioral change. Psychol Rev 1977:84:191-215.

49. Barry J, McQuade C, Livingstone T. Using nurse case management to promote self-efficacy in individuals with rheumatoid arthritis. Rehabil Nurs 1998·23:300-4.

50. Kallas KD. Establishing a self-administered medication program. J Nurs Adm 1984:14:38-42. 
51. Arvidsson SB, Petersson A, Nilsson I, et al. A nurse-led rheumatology clinic's impact on empowering patients with rheumatoid arthritis: a qualitative study. Nurs Health Sci 2006;8:133-9.

52. Larsson I, Arvidsson S, Bergman S, et al. Patients' perceptions of drug information given by a rheumatology nurse: a phenomenographic study. Musculoskeletal Care 2010;8:36-45

53. Gordon MM, Thomson EA, Madhok R, et al. Can intervention modify adverse lifestyle variables in a rheumatoid population? Results of a pilot study. Ann Rheum Dis 2002;61:66-9.

54. Temmink D, Hutten JB, Francke AL, et al. Rheumatology outpatient nurse clinics: a valuable addition? Arthritis Rheum 2001;45:280-6.

55. Grol R, Grimshaw J. Evidence-based implementation of evidence-based medicine. Jt Comm J Oual Improv 1999;25:503-13.

56. http://www.institute.nhs.uk/quality and service improvement tools/quality and service_improvement_tools/protocol_based_care.html laccessed 22 February 2011).

57. van Tuyl LH, Plass AM, Lems WF, et al. Facilitating the use of COBRA combination therapy in early rheumatoid arthritis: a pilot implementation study. $J$ Rheumatol 2009;36:1380-6.

58. Oliver S. Multidisciplinary disease management in rheumatology. Prof Nurse 2003; 19:137-41.

59. Voorneveld H, Duymaer van Twist L, Veldhuizen C. Development of a guideline for rheumatology nurses in the Netherlands about supervision, education and administer biological agents (abstract). Ann Rheum Dis 2008;67(Suppl II):670.

60. Hennell SL, Wood BB, Spark EW. Competency and the use of clinical management plans in rheumatology practice. Nurse Prescribing 2004;2:26-30.

61. El Miedany Y, Palmer D, El Gaafary M. Diagnosis of early arthritis: outcomes of a nurse-led clinic. Br J Nurs 2006;15:394-9.

62. Goh L, Samanta J, Samanta A. Rheumatology nurse practitioners' perceptions of their role. Musculoskeletal Care 2006:4:88-100.

63. Hehir M, Carr M, Davis B, et al. Nursing support at the onset of rheumatoid arthritis: time and space for emotions, practicalities and self-management. Musculoskeletal Care 2008;6:124-34.

64. Hill J, Oliver S, Finney D. Rheumatology nurses and health professionals in the UK: who are they and what do they do? (abstract). Ann Rheum Dis 2010;69(Suppl 3):744.

65. Hill J, Ryan S, Oliver S. The clinical practice and perceived competencies of rheumatology nurse specialists in the UK (abstract). Ann Rheum Dis 2010; 69(Suppl 3):725
66. Hurkmans EJ, Geenen R, Verhoef J, et al.Rheumatology health professionals perspectives on their current and future tasks and education (abstract). Ann Rheum Dis 2010;69(Suppl 3):89.

67. Long AF, Kneafsey R, Ryan J, et al. The role of the nurse within the multi-professional rehabilitation team. J Adv Nurs 2002;37:70-8.

68. Long AF, Kneafsey R, Ryan J. Rehabilitation practice: challenges to effective team working. Int J Nurs Stud 2003;40:663-73.

69. Oliver S, Leary A. Describing the complexity and value of the nurse specialist role in rheumatology in the UK using information technology (abstract). Ann Rheum Dis 2010;69(Suppl 3):717.

70. Juhola H, Kukkurainen ML, Suominen T. Rheumatology nurses' work-related empowerment. Musculoskeletal Care 2007:5:91-7.

71. Mäkeläinen P, Vehviläinen-Julkunen K, Pietilä AM. Rheumatoid arthritis patients' education—contents and methods. J Clin Nurs 2007:16:258-67.

72. Ryan S, Wylie E. An exploratory survey of the practice of rheumatology nurses addressing the sexuality of patients with rheumatoid arthritis. Musculoskeletal Care 2005;3:44-53.

73. Sierakowska M, Krajewska-Kulak E, Lewko J, et al. The education of patients with rheumatoid arthritis - the knowledge and expectation of patients - the opinions of rheumatology nurses. Rocz Akad Med Bialymst 2005;50(Suppl 1):107-10.

74. Laurant M, Reeves D, Hermens R, et al. Substitution of doctors by nurses in primary care. Cochrane Database Syst Rev 2005;2:CD001271.

75. Loveman E, Royle P, Waugh N. Specialist nurses in diabetes mellitus. Cochrane Database Syst Rev 2003:2:CD003286.

76. Craig P, Dieppe P, Macintyre S, et al. Developing and evaluating complex interventions: the new Medical Research Council guidance. BMJ 2008;337:a1655

77. Campbell $\mathbf{M}$, Fitzpatrick R, Haines A, et al. Framework for design and evaluation of complex interventions to improve health. BMJ 2000;321:694-6.

78. Ward V, Hill J, Hale C, et al. Patient priorities of care in rheumatology outpatient clinics: a qualitative study. Musculoskeletal Care 2007:5:216-28.

79. Oliver $\mathbf{S}$. Achieving quality care in rheumatoid arthritis. Practice Nursing 2009;20:460.

80. Esselens G, Westhovens R, Verschueren P. Effectiveness of an integrated outpatient care programme compared with present-day standard care in early rheumatoid arthritis. Musculoskeletal Care 2009;7:1-16. 